\title{
ON THE STRONG MAXIMUM PRINCIPLE FOR PARABOLIC DIFFERENTIAL EQUATIONS
}

\author{
by WOLFGANG WALTER
}

(Received 4th February 1985)

\section{Introduction}

In a recent paper [2], D. Colton has given a new proof for the strong maximum principle with regard to the heat equation $u_{t}=\Delta u$. His proof depends on the analyticity (in $x$ ) of solutions. For this reason it does not carry over to the equation

$$
u_{t}=\Delta u+c(t, x) u
$$

or to more general equations. But in order to tread mildly nonlinear equations such as $u_{t}=\Delta u+f(u)$ which are important in many applications, it is essential to have the strong maximum principle at least for equation $\left(^{*}\right)$. It should also be said that this proof uses nontrivial facts about the heat equation.

In what follows we give a simple proof valid for general linear parabolic equations which uses only some basic results from calculus.

\section{Basic facts about parabolic equations}

We consider the linear parabolic equation in $n$ space dimensions

$$
u_{t}=E u \equiv a u_{x x}+b u_{x}+c u
$$

where the abbreviations

$$
a u_{x x}=\sum_{i, j=1}^{n} a_{i j} u_{x_{i} x_{j}}, \quad b u_{x}=\sum_{i=1}^{n} b_{i} u_{x_{i}}, \quad x=\left(x_{1}, \ldots, x_{n}\right)
$$

have been used. The coefficients $a, b, c$ are assumed to depend on $(t, x)$ and the matrix $a=\left(a_{i j}\right)$ is assumed to be positive semidefinite, $\xi^{\top} a \xi \equiv \sum_{i, j=1}^{n} a_{i j} \xi_{i} \xi_{j} \geqq 0$ for $\xi \in \mathbb{R}^{n}$. Things are a little bit simpler if we restrict ourselves to the equation

$$
u_{t}=\Delta u+c u
$$

which is referred to as the "simple case". For simplicity, we consider a cylindrical domain,

$$
G=(0, T] \times D \text { and } \Gamma=\{0\} \times D \cup(0, T] \times \partial D,
$$


where $D \subset \mathbb{R}^{n}$ is open and bounded; $G$ is the "parabolic interior" and $\Gamma$ the "parabolic boundary" of $\bar{G}$. Let us remark that all results and proofs are also valid in the general case, where $\bar{G} \subset \mathbb{R}^{1+n}$ is compact. In this case, $G$ consists of all interior points of $\bar{G}$ and of those points $\left(t_{0}, x_{0}\right) \in \partial G$ for which a lower half-neighbourhood (consisting of those points $(t, x)$ of a neighbourhood for which $\left.t<t_{\mathrm{o}}\right)$ has only interior points, and $\Gamma=\bar{G} \backslash G$.

Let $Z$ be the class of functions $u$ continuous in $\vec{G}$ for which the derivatives $u_{t}, u_{x}=\left(u_{x_{i}}\right)$ and $u_{x x}=\left(u_{x_{i} x_{j}}\right)$ are continuous in $G$. The following result is needed.

(A) If $u \in Z$ has a local minimum with respect to $x$ at a point $(t, x) \in G$, then $a u_{x x} \geqq 0$ (or $\Delta u \geqq 0$ in the simple case) at this point.

In the simple case the proof is trivial, in the general case it uses (i) $u_{x x}$ is positive semidefinite at a local minimum, and (ii) if $a$ and $a^{\prime}$ are positive semidefinite matrices then $\sum a_{i j} a_{i j}^{\prime} \geqq 0$ (this is sometimes referred to as Schur's theorem).

First, we prove two basic facts about parabolic differential inequalities. It is assumed that $w \in Z$, but there are no regularity assumptions on $a, b, c$.

(B) $w_{t}>E w$ in $G, w>0$ on $\Gamma$ implies $w>0$ in $\bar{G}$.

(C) $w_{t} \geqq E w$ in $G, w \geqq 0$ on $\Gamma$ and $\sup _{G} c(t, x)<\infty$ implies $w \geqq 0$ in $\bar{G}$.

Proof. Assume that (B) is false. The set $N$ of all $(t, x) \in \bar{G}$ with $w(t, x) \leqq 0$ is compact, and there exists a "first" point $\left(t_{0}, x_{0}\right) \in N$, which means that $t \geqq t_{0}$ for all $(t, x) \in N$. Since $w>0$ on $\Gamma$, the point $\left(t_{0}, x_{0}\right)$ belongs to $G$, and because of continuity we have $w\left(t_{0}, x_{0}\right)=0$ and $w\left(t_{0}, x\right) \geqq 0$ for $x \in \bar{D}$. Hence $w\left(t_{0}, x\right)$ has a local minimum with respect to $x$ at $x_{0}$, and it follows that $a w_{x x} \geqq 0, b w_{x}=0$ and $c w=0$, i.e., $E w \geqq 0$ at $\left(t_{0}, x_{0}\right)$. Hence $w_{t}\left(t_{0}, x_{0}\right)>0$. But this inequality together with $w\left(t_{0}, x_{0}\right)=0$ implies that there are points $\left(t, x_{0}\right) \in G$ with $t<t_{0}$ where $w$ is negative. Hence a contradiction to the definition of $t_{0}$ is obtained, and $(\mathrm{B})$ is proved.

(C) is reduced to (B) by a simple trick. It follows from $c(t, x)<K$ that the function $\rho(t, x)=e^{K t}$ satisfies $\rho_{t}>E \rho$. Hence $w^{\varepsilon}=w+\varepsilon \rho(\varepsilon>0)$ satisfies $w_{t}^{\varepsilon}>E w^{\varepsilon}$ in $G$ and $w^{\varepsilon}>0$ on $\Gamma$. From (B) we get $w^{\varepsilon}>0$, which in turn leads to $w>0$ since $\varepsilon>0$ is arbitrary.

A simple consequence of $(C)$ is

(D) $v_{t} \leqq E v$ and $w_{t} \geqq E w$ in $G, v \leqq w$ on $\Gamma$ implies $v \leqq w$ in $\bar{G}$ if $\sup c<\infty$ and $v, w \in Z$.

This follows by applying (C) to $u:=w-v$.

We use the abbreviations $x y=x_{1} y_{1}+\cdots+x_{n} y_{n}, x^{2}=x x=|x|^{2}$ and $\operatorname{tr}(a)=a_{11}+a_{22}+\cdots$ $+a_{n n}$, in particular $b x=b_{1} x_{1}+\cdots+b_{n} x_{n}$. For a function $v(t, x)=\phi\left(x^{2}\right)$ the operator $E v$ is given, in terms of $\phi(s), s=x^{2}$, by

(E) $E v=4 x^{\top} a x \phi^{\prime \prime}(s)+2 \phi^{\prime}(s)(\operatorname{tr}(a)+b x)+c \phi$.

\section{The strong minimum principle}

Assume that $a, b, c$ are bounded and that $\xi^{\top} a \xi \geqq \delta \xi^{2}$ (strong ellipticity), where $\delta>0$. If 
$w \in Z$,

$$
w_{t} \geqq E w \text { in } G, \quad w \geqq 0 \text { on } \Gamma,
$$

then $w \geqq 0$ in $G$. If $w\left(t_{0}, x_{0}\right)=0$, where $x_{0} \in D$, then $w \geqq 0$ in $\left[0, t_{0}\right] \times D$.

Proof. The function $V(t, x)=e^{-A t}\left(R^{2}-x^{2}\right)_{+}^{3}(+$ indicates that $V=0$ for $|x|>R)$ is of class $C^{2}\left(\mathbb{R}^{n+1}\right)$. The inequality $V_{t} \leqq E V$ is obviously true for $x^{2}=s>R^{2}$. By $(\mathrm{E})$, this inequality is for $s<R^{2}$ equivalent to

$$
-A\left(R^{2}-s\right)^{3} \leqq 24 x^{\top} a x\left(R^{2}-s\right)-6\left(R^{2}-s\right)^{2}(\operatorname{tr}(a)+b x)+c\left(R^{2}-s\right)^{3},
$$

which in turn follows from

$$
(A+c)\left(R^{2}-s\right)^{2}+24 \delta s-6 K\left(R^{2}-s\right) \geqq 0,
$$

where $K$ is a bound for $|\operatorname{tr}(a)+b x|$ (note that $x^{\top} a x \geqq \delta s$ ). The last inequality is true for large $A$, since for $s$ close to $R^{2}$, say, for $R^{2}-\varepsilon \leqq s \leqq R^{2}$, the term $24 \delta s$ dominates the other terms, and for $0 \leqq s \leqq R^{2}-\varepsilon$ the last term is bounded and the first term can be made large, since $R^{2}-s \geqq \varepsilon>0$. Hence $V_{t} \leqq E V$ in $\mathbb{R}^{n+1}$.

Now let $v=v(t, x ; e):=V(t, x-t e)$, where $e \in \mathbb{R}^{n}$ is arbitrary. The inequality $v_{t} \leqq E v$ is equivalent to $V_{t} \leqq a V_{x x}+(b+e) V_{x}+c V$, hence it holds in $\mathbb{R}^{n+1}$ if in the above reasoning $b$ is replaced by $b+e$. Note that $v(t, x ; e)$ is positive in an infinite oblique cylinder with axis $(t, x)=t(1, e)(t \in \mathbb{R})$; this cylinder consists of all points $(t, x)$ with $|x-t e|<R$.

Now assume that $w(0, a)>0, a \in D$, and choose $R$ so small that $B_{R}(a) \subset D$ and $w(0, x)>0$ for $|x-a| \leqq R$. Then there exists $\varepsilon>0$ such that $\varepsilon V(0, x-a) \leqq w(0, x)$ in $D$. It follows from (D) that $\varepsilon V(t, x-a) \leqq w(t, x)$ and hence $w>0$ in $(0, T] \times B_{R}(a)$. The same reasoning can be carried out with $v=V(t, x-a-t e)$, where $e \in \mathbb{R}^{n}$ is arbitrary. If $T_{0} \leqq T$ is such that the oblique cylinder $0<t \leqq T_{0},|(t, x)-(t, t e)|<R$ lies in $G$, then $w>0$ in this cylinder. In particular, if the ball $B_{\rho}(a)$ is contained in $D$, then $w>0$ in $(0, T] \times B_{\rho}(a)$.

One can apply the same method of proof if, e.g., $w\left(t_{1}, a\right)>0$, where $t_{1}<t_{0}$. As a result, the inequality $w>0$ in $\left(t_{1}, T\right] \times B_{\rho}(a)$ is obtained. In order to show that $w\left(t_{0}, x_{0}\right)>0$ (this is the contradiction from which the strong maximum principle follows), one uses a chain of balls $B_{i}=B_{R_{i}}\left(\xi_{i}\right) \subset D(i=1, \ldots, p)$ with $\xi_{i+1} \in B_{i}, \xi_{1}=a, \xi_{p}=x_{0}$ and applies the above result in each ball. A similar chaining argument occurs in other instances, too, e.g., in the analytic continuation of holomorphic functions by power series.

Remarks. 1. In the case where $\bar{G}$ is an arbitrary compact set, the strong minimum principle is formulated in the following way: If $w\left(t_{0}, x_{0}\right)=0$, then $w(t, x)=0$ at every point of $G$, which can be connected with $\left(t_{0}, x_{0}\right)$ by a polygonal line (or continuous curve) along which $t$ is increasing. The above proof carries over to this case (with obvious changes).

2. Other forms of the strong minimum or maximum principle where $w \geqq \alpha$ or $w \leqq \alpha$ on the boundary are derived by considering $\bar{w}=w-\alpha$ or $\bar{w}=\alpha-w$, respectively. There are no problems when $c=0$, but in the general case the sign of $c$ plays a role. Assume, for 
example, that $w_{t} \geqq \Delta w+c w$ in $G, w \geqq \alpha$ on $\Gamma$. Then the strong minimum principle holds if $c \alpha \geqq 0$.

3. The mildly nonlinear case. Assume for simplicity that $u \in Z$,

$$
u_{t}=\Delta u+f(t, x, u) \text { in } G, u \leqq \alpha \text { on } \Gamma \text { and } f(t, x, \alpha)=0,
$$

where $f$ is Lipschitz continuous in $u$ in bounded subsets of $\bar{G} \times \mathbb{R}$. Then $u \equiv \alpha$ or $u<\alpha$ in $G$-for proof, one observes that, since $u \in Z$ is bounded,

$$
-f(t, x, u)=f(t, x, \alpha)-f(t, x, u)=c(t, x)(\alpha-u) \quad(u=u(t, x)),
$$

where $c$ is bounded. Hence $w=\alpha-u$ satisfies $w_{t}=\Delta w+c w$ in $G, w \geqq 0$ on $\Gamma$, which implies $w \equiv 0$ or $w>0$ by the strong minimum principle. In cases like this it is convenient to have the strong minimum principle at one's disposal without regularity assumptions on the coefficients. It follows at once from the Lipschitz property that a bounded function $c$ exists, but regularity properties are not so easily obtained.

4. The proof described here has been found by the author more than 20 years ago. It was first published in [3] (in connection with the nonlinear case which complicates matters somewhat); see also [4; p. 200f.]. Besala has used this method of proof in [1] (with quotation) for an extension of the strong maximum principle. In [5], Watson describes it as a "very simple method ... which seems to originate in [4]". Perhaps the general nonlinear setting of [4] has prevented this proof from becoming more widely known.

\section{REFERENCES}

1. P. Besala, An extension of the strong maximum principle for parabolic equations, Bull. Acad. Polon. Sci. Sér. Sci. Math. Astr. Phys. 19 (1971), 1003-1006.

2. D. Colton, The strong maximum principle for the heat equation, Proc. Edinburgh Math. Soc. 27 (1984), 297-299.

3. W. Walter, Differential- und Integral-Ungleichungen (Springer Tracts in Natural Philosophy, Springer-Verlag, 1964).

4. W. Walter, Differential and Integral Inequalities (Ergebnisse der Mathematik und ihrer Grenzgebiete, Vol. 55, Springer-Varlag, 1970) (enlarged translation of [3]).

5. N. A. Watson, The weak maximum principle for parabolic differential inequalities, Rend. Circ. Mat. Palermo, Serie II 33 (1984), 421-425.

Mathematisches Institut

UNIVERSITÃT. KARLSRUHE 